\title{
Measuring the Hedonic and Utilitarian Sources of Consumer Attitudes
}

\author{
RAJEEV BATRA \\ $4209 \mathrm{~F}$ Business Administration, School of Business Administration, University of Michigan, Ann \\ Arbor, MI 48109
}

OLLI T. AHTOLA

Tempere University, Finland

Key words: Attitudes, Preferences, Measurement

[Revised September 1990]

\begin{abstract}
It has been suggested theoretically that consumer attitudes have distinct hedonic and utilitarian components, and that product categories differ in the extent to which their overall attitudes are derived from these two components. This paper reports three studies that validate measurement scales for these constructs and, using them, show that these two attitude dimensions do seem to exist; are based on different types of product attributes; and are differentially salient across different consumer products and behaviors, in theoretically-consistent ways.
\end{abstract}

Overall consumer attitudes toward brands and consumption behaviors have typically been measured on a single evaluative dimension, often scaled using evaluative semantic differential (SD) scales identified by Osgood, Suci, and Tannenbaum (1957). It has recently been suggested in the consumer research literature, however, that consumer attitudes are inherently bidimensional, because consumers purchase goods and services and perform consumption behaviors for two basic reasons: (1) consummatory affective (hedonic) gratification (from sensory attributes), and (2) instrumental, utilitarian reasons concerned with "expectations of consequences" (of a means-ends variety, from functional and nonsensory attributes). (See Holbrook and Hirschman 1982; Millar and Tesser 1986; Triandis 1977). This paper tests this dimensionality empirically, and in the process validates measurement scales for these two subdimensions of overall attitudes.

The assumption of evaluative unidimensionality typically applies only to overall attitudes. When diagnostic insights into the composition of this overall attitude are sought, a multiattribute "adequacy-importance" (AI) model is often used, in which consumer ratings of the importance of different attributes are combined with ratings of the brand's adequacy on those attributes. Such compositional multiattribute data are usually factor analyzed to gain insight into the dimensionality of the attribute-based evaluation for that brand; these are sometimes called multidimensional AI models. However, while such analyses of multiattribute AI fac- 
tors aid in understanding a specific product category or consumer behavior, more general insights into the dimensionality of evaluations - applicable to all product categories or behaviors - are rarely available from such results.

Such across-category analyses of the dimensionality of overall attitudes might be useful for several reasons. First, they would tell us if consumers evaluate particular attributes along different evaluative dimensions: if there exist different types of attributes, and correspondingly different types of evaluations. While attribute typologies have been suggested before, they have focused more on scaling issues (e.g. Myers and Shocker 1983), and have not been empirically researched. Second, such across-category research could suggest appropriate promotional strategies for particular brands or behaviors. A well-known advertising planning model used by the Foote, Cone and Belding (FCB) agency (Vaughn 1986) classifies product categories on, among others, a thinking/feeling dimension, and suggests that while thinking products require more rational and informative advertising, feeling products are advertised more effectively using emotional and pleasure/ sensory appeals. Measuring these aspects of evaluative character might thus be useful for promotional strategy. Third, consumer behaviors may be better predicted by attitudes if the multi-item attitude measure used in such prediction averages across items from only the appropriate attitudinal subdimension, rather than across items from both subdimensions. Millar and Tesser (1986) showed that attitudes predict behaviors better when the evaluative basis for those attitudes matches the purpose of the behavior itself.

It would thus seem that the study of the hedonic and utilitarian dimensions of overall consumer attitudes merits empirical research, and therefore scale development and validation. Following well-known principles of measure validation (cf., Bagozzi 1980), we will seek below to develop measurement scales for these two dimensions - selected from among the evaluative SDs identified by Osgood et al (1957) - that not only have face validity but also display adequate (1) reliability (e.g., high internal consistency alpha coefficients, cf. Nunnally 1967, and high composite construct reliabilities, cf. Fornell and Larker 1981);

(2) convergent validity (high shared variance among multiple measures of each construct, relative to the amount of variance due to measurement error; measured through the "average variance extracted" statistic of Fornell and Larker 1981);

(3) discriminant validity (low agreement across the multiple measures of different constructs, measured through tests of the phi inter-construct correlations being less than 1.0, cf. Bagozzi 1980); and

(4) nomological validity (relationships across these two constructs that are consistent with theoretical expectations; e.g., that different brands and behaviors differ predictably in the extent to which overall attitudes are based on these two sources of evaluation). 


\section{Literature review}

\subsection{Theoretical review}

Before describing our scale development and validation efforts, it is necessary to briefly review the relevant theoretical literature. The authors cited earlier suggest that there exist two kinds of consumer evaluation, in which a consumption object is cognitively placed on both a utilitarian dimension of instrumentality (e.g., how useful or beneficial the object is), and on an hedonic dimension measuring the experiential affect associated with the object (e.g., how pleasant and agreeable those associated feelings are). Both of these types of benefits contribute, in differing degrees, to the overall goodness of a consumer good or behavior. These hedonic and utilitarian reasons or motivations for consumption need not be (and usually are not) mutually exclusive: a toothpaste may both prevent cavities and provide pleasure from its taste. Nor need these two motivations be evaluatively consistent: a consumption activity that gives me pleasure now may in fact be bad for me in an instrumental sense (e.g., smoking), while another that gives me no pleasure may in fact be instrumentally valuable (e.g., going to the dentist). Further, these two bases of evaluation may not be equally salient; some product categories, brands and behaviors may be more positively evaluated on one dimension than another, and different objects should differ predictably in the extent to which overall attitudes towards them are hedonic or utilitarian. A behavior performed for hedonic or fun reasons, for example, should have its overall attitudinal evaluation based relatively more on that antecedent. In the terminology we will use here, the hedonic determinant of overall evaluations is presumed to be based on the consumer's assessment of how much pleasure he gets; his utilitarian determinant is based on his assessment about the instrumental value of the brand's functional attributes. For convenience, both kinds of assessments will be modeled here in the traditional AI framework; this is not meant to be a representation of the actual information processing steps involved.

\subsection{Empirical support from prior studies}

In nonconsumer domains, several studies that have restricted themselves to examining the factor structure of attitudes within a battery of evaluative SD items have, in fact, succeeded in finding evidence for different kinds of evaluation (e.g. Komorita and Bass 1967; Levin 1965; Osgood, Suci and Tannenbaum 1957, p. 77, p. 187, p. 326). Several of these studies have found differential evaluative salience of the kind of attitude across different types of attitude stimuli: depending on the stimulus, a different attitudinal factor - on which the good-bad item loads - might dominate in an overall good-bad assessment. However, no systematic empirical research on the dimensionality of SD attitudes has yet been reported in the consumer research literature. 


\subsection{Selection of measurement scales for validation}

The SD scales used in the studies below all form part of the battery of items identified by Osgood, Suci and Tannenbaum (1957) as being attitudinal or evaluative. Prior literature indicates that it is indeed possible to measure multiple attitude dimensions using such SD scales. In addition to the previously cited results of Komorita and Bass (1967), we also relied on Triandis (1977). He has argued that behavioral intentions are a function not of a unidimensional attitude construct, but instead of expectations of consequences (measured on items like valuable-harmful) and of affect (e.g., enjoyable, interesting, and pleasant.)

With this prior research as background, we now report studies that seek to develop scales for the hedonic and utilitarian dimensions that possess adequate (a) reliability, (b) convergent validity, (c) discriminant validity, and (d) nomological validity. Using these scales, we will show that while attitudes towards different products and different behaviors will each show these two SD factors (hedonic and utilitarian), the two factors will differ (across products and behaviors, in theoretically-consistent ways) in the degree to which they are based on different kinds of product attributes, and to which they influence overall attitudinal judgments (e.g., good-bad). By theoretically consistent, we mean (1) that hedonic attitudes will be based on AI evaluations of sensory attributes (e.g., taste) and utilitarian attitudes on AI assessments of functional attributes (e.g., decay prevention), and (2) that products and behaviors that are primarily fun ones (e.g., attending a rock concert) will have the hedonic component dominating overall evaluation, while those that are primarily functional (e.g., going to a dentist) will have a dominant utilitarian component.

\section{Study 1}

Sixteen 7-point evaluative SD items from the battery used by Osgood, Suci, and Tannenbaum (1957) were used in this study, selected judgmentally to ensure adequate coverage of the hedonic and utilitarian domains. Fifty-nine respondents rated four arbitrarily-selected brands (Pepsi, Listerine, Comet cleanser, and Cadillac) on each item.

Initial analysis consisted of (exploratory) common factor analysis for the four brands (a) individually and (b) pooled together (for generality). In almost all cases, a two-factor structure emerged: one factor, labeled hedonic, loaded heavily on pleasant-unpleasant, agreeable-disagreeable, nice-awful, and similar items; the second factor, labeled utilitarian, loaded heavily on useful-useless, beneficialharmful, important-unimportant and similar items. Interestingly, though no separate overall attitude factor emerged in these data, items we would expect to indicate overall attitudes (such as good-bad, positive-negative) loaded about equally on both factors for the pooled data (see Table 1) for Cadillac and Listerine. However, for Pepsi they load highest on the hedonic factor, and for Comet on the 
Table I. Study 1: Affective factor structure: (all four brands merged)

\begin{tabular}{|c|c|c|c|c|}
\hline \multicolumn{3}{|c|}{$\begin{array}{r}\text { (Common factor, total variance (of } 2 \text { factors, Ei } \\
\text { Orthogonal } \\
\text { (varimax) }\end{array}$} & \multicolumn{2}{|c|}{$\begin{array}{l}=91.8 \% \text { ) } \\
\quad \text { Oblique } * \\
\text { (factor pattern) }\end{array}$} \\
\hline & $\begin{array}{l}\text { Factor } \\
\# 1\end{array}$ & $\begin{array}{l}\text { Factor } \\
\# 2\end{array}$ & $\begin{array}{l}\text { Factor } \\
\# 1\end{array}$ & $\begin{array}{l}\text { Factor } \\
\# 2\end{array}$ \\
\hline Unrotated variance explained $\%$ & 75.4 & 16.4 & & \\
\hline Pleasant-Unpleasant & 0.852 & 0.188 & 0.922 & 0.061 \\
\hline Agreeable-Disagreeable & 0.806 & 0.427 & 0.852 & -0.206 \\
\hline Nice-Awful & 0.753 & 0.396 & 0.757 & -0.192 \\
\hline Harmonious-Dissonant & 0.593 & 0.196 & 0.640 & -0.026 \\
\hline Sociable-Unsociable & 0.515 & -0.063 & 0.543 & 0.220 \\
\hline Positive-Negative & 0.645 & 0.510 & 0.626 & -0.345 \\
\hline Like-Dislike & 0.583 & 0.468 & 0.544 & -0.321 \\
\hline Good-Bad & 0.544 & 0.628 & 0.487 & -0.502 \\
\hline Useful-Useless & 0.143 & 0.795 & 0.006 & -0.803 \\
\hline Beneficial-Harmful & 0.044 & 0.704 & -0.035 & -0.731 \\
\hline Important-Unimportant & 0.211 & 0.684 & 0.078 & -0.666 \\
\hline Meaningful-Meaningless & 0.159 & 0.563 & 0.009 & -0.556 \\
\hline Intelligent-Unintelligent & 0.324 & 0.489 & 0.264 & -0.422 \\
\hline
\end{tabular}

*After rotation with Kaiser normalization

utilitarian factor. This suggests that a good cleanser is one that is superior on utilitarian (e.g., useful, beneficial) aspects, while a good soft drink is one that is superior on hedonic (e.g., pleasant, nice) dimensions, and supports the nomological validity of the hedonic/utilitarian distinction.

The three highest-loading items from each factor in the exploratory factor analysis were next analyzed through confirmatory factor analysis for convergent and discriminant validity (Joreskog and Sorbom 1985; Bagozzi 1980). In this selection, we deliberately excluded the overall attitudinal items which displayed high concept $x$ scale interaction; we also relied on additional confirmatory factor analysis, which showed that these were also the best-fitting six items after iterative itemdeletion. One and Two Factor models were compared using the incremental goodness-of-fit indices suggested by Bentler and Bonett (1980), for the four brands individually as well as pooled. The index for the two-factor model was always greater than 0.92 (conventional cut-off: 0.90 ), and was always greater than the index for the one-factor model, suggesting that a two-factor model fits the data better. The average variance extracted, a measure of the variance captured by each construct (relative to measurement error; see Fornell and Larker 1981, p. 46) was 0.77 for the hedonic component, and 0.55 for the utilitarian (pooled data), above the minimum of 0.50 conventionally used to establish convergent validity. Composite construct reliabilities (Fornell and Larker 1981, p. 45) were generally high ( 0.91 for all brands) for the hedonic factor, but a little lower $(0.78$ for all 
brands) for the utilitarian factor, suggesting that the hedonic factor was more clearly defined in these data than the utilitarian.

In the test for discriminant validity, the inter-factor correlation (phi) was significantly below 1.0, thus establishing significant unique (unshared) variance for the two factors (chi-square difference significant at $p<.05$ for models with and without phi constrained to unity). For the pooled brands data, this phi coefficient was only 0.547 (standard error $=0.057$ ), and the average variance extracted for each factor $(0.77$ and 0.55$)$ was well above the square of this phi $(0.30)$, again supporting discriminant validity (see Fornell and Larker 1981, p. 46). The two-factor model thus achieves both convergent and discriminant validity using commonly used tests, while the one factor model fails.

\section{Study 2}

The primary objective of this second study was to see if the hedonic and utilitarian scales related as theoretically predicted to AI composites created from independently-rated hedonic or utilitarian brand attributes. One hundred and eight student subjects saw an ad for a new toothpaste brand (toothpastes were used because they have both utilitarian and hedonic benefits). They then provided data on brand attitudes; the reasons why they bought toothpastes (rational or emotional/feel good/pleasure), and adequacy-importance measures of the brand (on 7 attributes). The analyses reported are limited to those subjects ( $80 \%$ of the sample) who had not tried the brand before. (Analyses using all subjects yielded similar results).

Brand attitudes were measured through 9 evaluative SDs: pleasant-unpleasant, useful-useless, good-bad, positive-negative, worthless-valuable, unfavorablefavorable, disagreeable-agreeable, harmful-beneficial, and dislike-like. Again, two factors emerged in Varimax-rotated principal components analysis. Items loading most heavily on the first (apparently hedonic, but also somewhat overall) factor were favorable-, agreeable-, positive-, like-, good-, and pleasant-; items on the second (utilitarian) factor were beneficial-, valuable-, and useful-.

A one-factor model with nine items (six highest from the first factor, three highest from the second) failed to reach convergent validity (chi-square with $9 \mathrm{df}=$ $53.87, \mathrm{p}<.001$, incremental fit index $=0.85$ ) while a two-factor model with the appropriate items did so (chi-square with $8 \mathrm{df}=5.94, \mathrm{p}<.654$, incremental fit index $=0.95$ ). The two-factor model also achieved discriminant validity (chisquare difference with $1 \mathrm{df}=8.26$ ). Scales for the two SD factors were then created, using beneficial-, valuable-, and useful- for the utilitarian component, and pleasant-, agreeable-, and like- for the hedonic component (thus excluding those items that, theoretically and in other studies, appeared to be measuring overall attitudes). Each scale had a coefficient alpha above 0.84 .

Nomologically, if these two SD attitude factors are indeed measuring what they should theoretically, the hedonic attitude subdimension should relate more 
strongly to AI evaluations of sensory fun attributes, and the utilitarian attitude subdimension should relate more strongly to instrumental, means-ends attributes. Principal components analysis of the attribute adequacy ratings yielded two major factors, the first loading on whiteness, decay and plaque/tartar, presumably more instrumental, means-ends attributes, and the second on freshens mouth and taste/ flavor, presumably attributes providing sensory, affective gratification. (Analyses of the attribute importance ratings, and the AI product-terms, also yielded essentially similar factors, but their two major factors explained less of the variance, and were thus not pursued further.)

Based on the factor loadings just described, scales were created for instrumental attribute adequacy (plaque/tartar, decay, whiteness; alpha 0.79) and for sensory attribute adequacy (mouth freshness, taste/flavor; alpha 0.89). Correlations were then computed between these two attribute adequacy scales and the two attitude sub-dimension scales. Since the two attribute adequacy scales themselves correlated highly $(r=0.61)$, partial rather than zero-order correlations were used, controlling for the attribute adequacy scale not being related in each attributeadequacy/attitude correlation. These partial correlations showed that the hedonic attitude SD scale correlates more strongly than does the utilitarian attitude SD scale with the sensory attribute adequacy scale $(r=.45$ vs. $.16, z=5.65$, $\mathrm{p}<.05$ ), while the utilitarian attitude SD scale correlates more strongly than the hedonic SD scale with the instrumental attribute adequacy scale $(r=.36 \mathrm{vs} . .01$, $z=6.34, p<.001$ ). Thus the two SD attitude components appear to possess not only convergent and discriminant validity, but they also appear to be measuring what they are supposed to, and thus possess nomological validity.

Based on the conceptualization of the two components, we would expect that consumers buying toothpastes for rational reasons would place greater overall evaluative weight on the utilitarian attitude SD component, while those buying it for emotional/feel good/pleasure reasons would place greater evaluative weight on the hedonic attitude SD component. Recall that each subject rated his/her reason to buy toothpastes on two scales (rational, or emotional/feel good/pleasure). To test the differential weight hypothesis, each subject was now placed into a high (or low) rational reason-to-buy group, and a high (or low) emotional/feel good/ pleasure reason-to-buy group, using median splits on these two reason-to-buy scales. Then, separately for each of the two high subsamples, the hedonic and utilitarian SD attitude scales were used to predict the overall good-bad SD item via multiple regression. As would be predicted nomologically, for the high emotional/feel good/pleasure reasons subsample the hedonic SD attitude scale was a much stronger predictor of overall attitudes than the utilitarian SD scale (betas $0.80[\mathrm{p}<.01]$ vs. 0.06 [n.s.] respectively), while the situation was reversed in the high rational reasons subsample (betas 0.33 [p $<.03]$ vs. $0.54[p<.01]$ respectively).

Importantly, this shifting basis of overall goodness might not always be apparent by simply asking subjects to provide importance ratings of the different attributes. When the attribute importance ratings obtained in this study were com- 
pared across the different reasons-to-buy median-split subsamples, no significant differences emerged on these important ratings. In all, subjects rated decay prevention the most important, followed by removing plaque/tartar. It would appear that in such compositional assessments subjects seek to appear more utilitarian than they actually are.

\section{Study 3}

Data for this third study were collected by the second author without knowledge of the first two studies; as a result, the items used here do not correspond exactly to those used in those studies. Nonetheless, these data allow us to perform additional analyses examining the contribution of the two attitude components to overall attitudes in a different domain (behaviors, rather than brands), thus adding to the evidence on validity. Ninety-three introductory marketing students rated 18 behaviors on each of 23 evaluative SD items (the behaviors and the items are listed below). While some of the behaviors were expected a priori to be performed more for sensory gratification, such as going to a bar or rock concert, others were expected to be low on such positive sensory gratification but higher on instrumental, expectations of consequences value, such as going to the dentist or doing laundry.

To test whether the results from Study One and Two would replicate, the SD data for each stimulus were initially analyzed using principal components analysis. A varimax-rotated two-factor solution was extracted in each case, for consistency with those studies, and always explained at least $60 \%$ of the variance. Summary results are in Table 2. They show, for each of the 23 items, the number of times (for the 18 analyses) that each item had a loading greater than or equal to 0.50 on the hedonic $(\mathrm{H})$ or utilitarian $(\mathrm{U})$ factor, as well as the number of times the scale failed to have such a loading on either factor $(\mathrm{O})$ or had such a loading on both factors (HU).

It can be seen that several items (e.g., pleasant, beautiful, pleasing, happy, interesting, comfortable, and soothing) are almost always hedonic, while others (e.g., valuable, wise, safe, ordered, sane) are almost always utilitarian. These data suggest that while SD attitude measures do indeed suffer from concept $x$ scale interaction, as previous research (e.g., Levin 1965) has demonstrated, many of them load consistently enough on the hedonic or utilitarian factors across concepts to justify their use in research on these two attitude components. Note also that there are certain items (e.g., positive, good, favorable, and rewarding) that load most often on both factors, thus apparently reflecting what we would call overall attitudes.

Confirmatory analyses on the pooled data showed that the four highest loading utilitarian items were valuable-worthless, wise-foolish, meaningful-meaningless, and safe-dangerous. Of these, at least the first two are clearly utilitarian, if wise is interpreted as implying something sensible and prudent, having beneficial con- 
Table 2. Study 3: Summary of factor loadings

\begin{tabular}{lcccc}
\hline & Hedonic $(\mathrm{H})$ & Utilitarian $(\mathrm{U})$ & Neither $(\mathrm{O})$ & Both $(\mathrm{HU})$ \\
\hline 1. Pleasant-Unpleasant & $17^{*}$ & 0 & 1 & 0 \\
2. Meaningful-Meaningless & 7 & 8 & 3 & 0 \\
3. Successful-Unsuccessful & 9 & 8 & 2 & 2 \\
4. Beautiful-Ugly & 17 & 1 & 1 & 1 \\
5. Valuable-Worthless & 6 & 11 & 1 & 0 \\
6. Wise-Foolish & 6 & 14 & 0 & 2 \\
7. Positive-Negative & 11 & 12 & 0 & 5 \\
8. Good-Bad & 10 & 12 & 0 & 4 \\
9. Pleasing-Annoying & 18 & 0 & 0 & 0 \\
10. Clean-Dirty & 8 & 4 & 6 & 0 \\
11. Safe-Dangerous & 6 & 11 & 2 & 1 \\
12. Happy-Sad & 18 & 1 & 0 & 1 \\
13. Ordered-Chaotic & 2 & 12 & 4 & 0 \\
14. Smooth-Rough & 9 & 7 & 2 & 0 \\
15. Profound-Superficial & 0 & 7 & 11 & 0 \\
16. Rational-Emotional & 0 & 13 & 5 & 0 \\
17. Interesting-Boring & 17 & 1 & 0 & 0 \\
18. Comfortable-Uncomfortable & 18 & 1 & 0 & 1 \\
19. Soothing-Aggravating & 17 & 1 & 0 & 0 \\
20. Sane-Insane & 1 & 17 & 1 & 1 \\
21. Reputable-Disreputable & 5 & 10 & 4 & 1 \\
22. Favorable-Unfavorable & 13 & 8 & 0 & 3 \\
23. Rewarding-Punishing & 9 & 8 & 2 & 1 \\
\hline
\end{tabular}

*Figures represent no. of times (out of 18 ) that this factor loading was $\geqslant 0.50$.

sequences later even if not giving pleasure now. Scale reliabilities (alphas) were over 0.75 for 14 of the 18 behaviors. For the hedonic scale, the top five were pleasing-annoying, pleasant-unpleasant, comfortable-uncomfortable, happy-sad, and soothing-aggravating. These are clearly hedonic; since the first two are similar, pleasant-unpleasant was dropped in favor of soothing-aggravating to develop a four-item scale (alphas over 0.80 for 15 of the 18 behaviors).

Convergent validity estimates (average variance extracted statistics) for the utilitarian component exceeded 0.60 for 13 of the 18 behaviors, and were over 0.53 in all cases ( 0.50 is the conventional minimum). For the hedonic component, it always exceeded 0.52 , being greater than 0.60 for 17 of the 18 behaviors. For discriminant validity, the phi inter-construct correlation coefficient should be significantly less than unity. This was satisfied (at $p<.05$ ) in 13 of the 18 cases. It was not met in five: drinking milk; having beer with friends; going to a rock concert with friends; smoking a cigarette after meals, and having a drink when depressed. One could speculate that these five are cases where the utility of the behavior is perceived to be nothing more than its hedonic value, so that the correlation between the two factors (as measured) is consequently excessively high. LISREL was also used to estimate a model in which the two components (cor- 
related among themselves) predicted overall (general) attitudes, using the items for each construct just discussed. The model and specific items appeared to fit well for most specific behaviors: the goodness of fit index exceeded 0.80 in 15 of the 18 cases (the other three were $0.74,0.76$, and 0.76 ).

The standardized coefficients obtained for the hedonic and utilitarian latent variables as predictors of the general attitude latent variable (the gammas) indicate the relative influence of these two components on overall attitudes. For those behaviors where the phi coefficient between the two components are low (e.g., below 0.65 ), the gammas can be interpreted without much fear of multicollinearity. Using this cutoff, the results suggest that the behaviors of brushing teeth, doing the laundry, skipping classes when tired, taking vitamins, having dental check-ups, and setting apart daily study time, are all activities in which overall attitudes are influenced relatively more by the utilitarian than the hedonic component, with the utilitarian gamma always over 0.71 and the hedonic gamma always below 0.25 in these cases. Conversely, test driving a Mercedes Benz is seen as predominantly influenced by the hedonic component, with a hedonic gamma of 0.73 higher than the utilitarian gamma of 0.41 . Certain other behaviors, such as going to a hairdresser/barber, and eating in an expensive restaurant, are both about evenly hedonic and utilitarian.

In general, though the patterns found empirically in this study had not been predicted on the basis of some other external assessment, they seem to be supportive of the validity of the scales being developed. When measured on these scales, stimuli (in this case, behaviors) vary intuitively in the degree to which their overall attitudinal assessments are based on hedonic or utilitarian criteria, with those we would a priori label more functional having higher gammas for the utilitarian component. Unfortunately, those behaviors that might otherwise be expected to be predominantly hedonic (such as having a beer with friends, or going to a rock concert) have highly correlated attitude components, which leads to inadequate discriminant validity and makes their gammas difficult to interpret.

\section{General discussion}

The studies reported here quite clearly indicate that attitudes towards brands and behaviors have at least two distinct components, hedonic and utilitarian. A unidimensional model of SD attitudes failed to achieve convergent validity, while models with hedonic and utilitarian components achieved both convergent and discriminant validity. The studies also demonstrated nomological validity: the scales behaved as the theoretical constructs were intended to, in relating to product attributes and product characteristics. Study One showed that a brand like Comet cleanser is seen as good if it scores high on utilitarian aspects, while one like Pepsi is good based on the hedonic factor. Study Two showed that while the hedonic component is associated with sensory, experiential product attributes, the utilitarian one is associated with more instrumental, functional attributes. Further, the reasons for which a toothpaste was purchased by different benefit seg- 
ments determined which of these two attitudinal dimensions was more important in determining overall evaluation. The third study suggested that behaviors performed largely because they are beneficial for you have overall attitudes in which the utilitarian dimension is particularly salient, while behaviors that have higher intrinsic levels of fun and self-indulgence have overall attitudes in which the hedonic dimension has higher salience (though interpretation of some of these results were clouded by potential multicollinearity).

The contribution of this paper was to test if the theoretical notion of these two components was supported in consumer data, and to suggest scales that can be used to measure the hedonic and utilitarian components reliably and validly. Though the scales used varied slightly across the three studies, the cumulative evidence suggests to us that the utilitarian component can most validly be measured by the SD items of useful/useless, valuable/worthless, beneficial/harmful, and wise/foolish, and the hedonic component by the items pleasant/unpleasant, nice/awful, agreeable-disagreeable, and happy/sad. It would appear that SD items of good/bad, positive/negative, like/dislike and favorable/unfavorable most clearly measure overall attitudes, but that they can sometimes load on the hedonic factor. These results thus provide empirical support to theoretical ideas that have appeared earlier in the literature, and suggest scales for future research.

Clearly, such scale development needs to continue. Future development ought to use split-half validation procedures. Further, despite the evidence of Table 2, concept $x$ scale interaction remains a potential problem that must be minimized. Theoretically, given the nature of the differential attribute linkages shown in the second study, it does appear to make sense to label our two components hedonic and utilitarian. However, this does not mean that no interpretational ambiguities remain, and these require resolution. In particular, work is needed on the relationship, if any, between the hedonic and utilitarian constructs studied here and what the literature in cognitive social psychology has long labeled affective and cognitive attitudes. Research is also required on other attitude components (such as social or normative value, cf. Sirgy 1982) that are probably relevant for some consumer products or behaviors and may not be fully captured in the scales developed here.

Using such scales, researchers modeling consumer attitudes should attempt to measure multidimensional attitudes, and empirically explore possible implications of such multidimensionality for the development of attribute taxonomies, the construction of decompositional preference models, the prediction of behavioral intentions, and the better understanding of product category "character," for use in promotional and other managerial decisions.

\section{References}

Bagozzi, Richard P. (1980). Causal Models in Marketing. New York: Wiley.

Bentler, P. M., and D. G. Bonett. (1980). "Significance Tests and Goodness of Fit in the Analysis of Covariance Structures," Psychological Bulletin 24, 163-204. 
Fornell, C., and Larker, D. F. (1981). "Evaluating Structural Equation Models with Unobservable Variables and Measurement Error," Journal of Marketing Research 18, 39-50.

Holbrook, M. B. and E. C. Hirschman. (1982). "The Experiential Aspects of Consumption: Consumer Fantasies, Feelings, and Fun," Journal of Consumer Research 9, 132-140.

Joreskog, Karl G., and Dag Sorbom. (1985). LISREL VI: Analysis of Linear Structural Equations by Maximum Likelihood, Instrumental Variables and Least Squares Methods. Mooresville, IN: Scientific Software.

Komorita, S. S., and A. R. Bass. (1967). "Attitude Differentiation and Evaluative Scales of the Semantic Differential," Journal of Personality and Social Psychology 6, 241-244.

Levin, Joseph. (1965). "Three-Mode Factor Analysis," Psychological Bulletin 64 (6), 442-452.

Millar, M. G., and Tesser, A. (1986). "Effects of Affective and Cognitive Focus on the AttitudeBehavior Relationship." Journal of Personality and Social Psychology 51, 270-276.

Myers, James H., and Allan D. Shocker. (1981). "The Nature of Product-Related Attributes," in J. N. Sheth (ed.), Research in Marketing, Vol. V. Greenwich, CT: Jai Press, 211-236.

Osgood, C. E., G. J. Suci, and P. H. Tannenbaum. (1957). The Measurement of Meaning. Urbana, IL: University of Illinois Press.

Sirgy, M. J. (1982). "Self-Concept in Consumer Behavior: A Critical Review," Journal of Consumer Research 9(3): 287-300.

Triandis, H. C. (1977). Interpersonal Behavior. Monterey, CA: Brooks/Cole Publishing Co.

Vaughn, Richard. (1986). "How Advertising Works: A Planning Model Revisited," Journal of Advertising Research 26 (1), 57-66. 